\title{
EQUIVARIANT AFFINE LINE BUNDLES AND LINEARIZATION
}

\author{
Hanspeter Kraft and Frank Kutzschebauch
}

\begin{abstract}
A Bstract. We show that every algebraic action of a linearly reductive group on affine $n$-space $\mathbb{C}^{n}$ which is given by Jonquière automorphisms is linearizable. Similarly, every holomorphic action of a compact group $K$ by (holomorphic) Jonquière automorphisms is linearizable. Moreover, any holomorphic action of $K$ on $\mathbb{C}^{2}$ by overshears is linearizable, too. These results are based on the fact that equivariant algebraic or holomorphic affine line bundles over $\mathbb{C}^{n}$ are trivial.
\end{abstract}

\section{$\S 1$. Algebraic affine line bundles}

Let $G$ be an algebraic group and let $X$ be a variety with an algebraic action of $G$. We assume that the base field $k$ is algebraically closed of arbitrary characteristic (cf. Remark 3 at the end of $\S 1$ ). Consider the trivial affine line bundle $\pi: X \times \mathbb{A}^{1} \rightarrow X$ over $X$. We want to study the possible lifts of the $G$-action to $X \times \mathbb{A}^{1}$. Clearly, every character $\chi$ of $G$ defines such a lift:

$$
g(x, z):=(g x, \chi(g) \cdot z) \text { for } g \in G, x \in X, z \in \mathbb{A}^{1} .
$$

We call this a trivial lift (with character $\chi$ ).

Proposition 1. Assume that $G$ is linearly reductive and that $G$ acts trivially on the invertible regular functions on $X$. Then every lift of the $G$ action is equivalent to a trivial lift.

As usual, we denote by $\mathcal{O}(X)$ the (global) regular functions on $X$ and by $\mathcal{O}(X)^{*}$ the invertible ones.

Remark 1 . The assumption about the action on the invertible functions on $X$ is always satisfied if $\mathcal{O}(X)^{*}=k^{*}$ or if $G$ is connected (see [KK89, $§ 1$, Proposition 1.3]). However, the assumption is necessary as shown by the

Received July 11, 1996.

Both authors were partially supported by SNF (Schweizerischer Nationalfonds). 
following example. Let $G=\mathbb{Z} / 2 \mathbb{Z}$ act on $X:=\mathbb{A}^{1} \backslash\{0\}$ by $x \mapsto x^{-1}$. Then the lift to $X \times \mathbb{A}^{1}$ given by $(x, z) \mapsto\left(x^{-1}, x z\right)$ is not equivalent to a trivial lift since the representations on the fibers over the two fixed points 1 and -1 are not equivalent.

As an easy consequence of Proposition 1 we get the following result. (The special case where $G$ is a finite cyclic group was proved by Ivanenko [Iv96].)

Corollary 1. Let $G$ be a linearly reductive group acting algebraically on affine $n$-space $\mathbb{A}^{n}$ by Jonquière automorphisms. Then the action is linearizable, i.e., the image of $G$ in Aut $\mathbb{A}^{n}$ is conjugate to a subgroup of the diagonal matrices.

Proof. Recall that the Jonquière subgroup $\mathfrak{J}_{n}$ is the following subgroup of polynomial automorphisms of $\mathbb{A}^{n}$ :

$$
\begin{array}{r}
\mathfrak{J}_{n}:=\left\{\varphi=\left(\varphi_{1}, \varphi_{2}, \ldots, \varphi_{n}\right) \in \operatorname{Aut} \mathbb{A}^{n} \mid \varphi_{i} \in k\left[x_{1}, x_{2}, \ldots, x_{i}\right]\right. \\
\text { for } i=1, \ldots, n\}
\end{array}
$$

If the action of $G$ is by automorphisms from $\mathfrak{J}_{n}$ then we get induced $G$ actions on the subspaces $\mathbb{A}^{j} \subset \mathbb{A}^{n}$ of the first $j$ coordinates, and the corresponding projections

$$
\mathbb{A}^{n} \rightarrow \mathbb{A}^{n-1} \rightarrow \mathbb{A}^{n-2} \rightarrow \cdots \rightarrow \mathbb{A}^{2} \rightarrow \mathbb{A}^{1}
$$

are $G$-equivariant. By induction, we can assume that the action is linear on $\mathbb{A}^{n-1}$. Then the claim follows from Proposition 1 applied to the affine line bundle $\mathbb{A}^{n} \rightarrow \mathbb{A}^{n-1}$.

Proof of Proposition 1. The $G$-action on the bundle $X \times \mathbb{A}^{1}$ has the form

$$
g(x, z)=\left(g x, \lambda_{g}(x) z+\mu_{g}(x)\right)
$$

where $\lambda$ and $\mu$ are regular functions on $G \times X$ satisfying

$$
\begin{aligned}
& \lambda_{g h}(x)=\lambda_{g}(h x) \cdot \lambda_{h}(x), \quad \lambda_{e}(x) \equiv 1 \\
& \mu_{g h}(x)=\lambda_{g}(h x) \cdot \mu_{h}(x)+\mu_{g}(h x), \quad \mu_{e}(x) \equiv 0
\end{aligned}
$$

We first claim that $\lambda$ does not depend on $x \in X$. Since for any $g \in G$ the function $\lambda_{g}(x)$ is invertible it follows from the assumption that $\lambda_{g}(h x)=$ $\lambda_{g}(x)$ for all $h \in G$. Thus, by (a), $\lambda_{g}(x)$ is a character of $G$, for every $x \in X$. If $G$ is connected then the invertible function $\lambda$ on $G \times X$ has the form $\lambda_{g}(x)=\chi(g) \cdot f(x)$ where $\chi \in \mathcal{O}(G)$ and $f \in \mathcal{O}(X)$ (see [KK89, 11 
Proposition 1.1]). Since $\lambda_{e}(x) \equiv 1$ we see that $f$ is a constant and so $\lambda$ is independent of $x \in X$. In general, we have $g^{n} \in G^{0}$ for some $n \in \mathbb{N}$. Hence, $\lambda_{g}(x)^{n}=\lambda_{g^{n}}(x)$ is independent of $x$, and the claim follows.

It is clear now that the action on $X \times \mathbb{A}^{1}$ is equivalent to a trivial lift (with character $\lambda$ ) if and only if the bundle $\pi: X \times \mathbb{A}^{1} \rightarrow X$ has a $G$ equivariant section $s: X \rightarrow X \times \mathbb{A}^{1}, x \mapsto(x, \sigma(x))$. This means that the regular function $\sigma \in \mathcal{O}(X)$ satisfies

$$
\sigma(g x)=\lambda_{g} \cdot \sigma(x)+\mu_{g}(x) \quad \text { for } g \in G, x \in X .
$$

Now we consider the following locally finite $G$-representation on $\mathcal{O}(X)$ :

$$
g * f(x):=\lambda_{g} \cdot f\left(g^{-1} x\right) \quad \text { for } g \in G, x \in X \text {. }
$$

(This is the regular representation on $\mathcal{O}(X)$ multiplied with the character $\lambda$.) Define $\rho \in \mathcal{O}(G \times X)$ by

$$
\rho_{g}(x)=\lambda_{g} \cdot \mu_{g^{-1}}(x)
$$

and consider it as a map $G \rightarrow \mathcal{O}(X)$. Then equation (b) becomes

$$
\rho_{g h}=\rho_{g}+g * \rho_{h}
$$

i.e., $\rho: G \rightarrow \mathcal{O}(X)$ is an "algebraic" cocycle. (A map $\eta: Y \rightarrow \mathcal{O}(Z)$ where $Y, Z$ are algebraic varieties is called algebraic if the corresponding function $\eta(y, z):=\eta(y)(z)$ on $Y \times Z$ is regular.) Moreover, the third equation (c) becomes

$$
\rho_{g}=g * \sigma-\sigma
$$

This means that the lift is equivalent to a trivial lift if and only if $\rho$ is a coboundary. But it is well known that for a linearly reductive group $G$ every algebraic cocycle with values in a (locally finite and rational) representation is a coboundary:

$$
H_{\text {alg }}^{1}(G, M)=0 \quad \text { for every } G \text {-module } M \text {. }
$$

(We will give a short argument for this claim in Remark 2 below.) This proves our result.

Remark 2. The proof above shows that for a given $G$-variety $X$ all lifts of the $G$-action to $X \times \mathbb{A}^{1}$ are equivalent to trivial lifts if and only if we have $H_{\text {alg }}^{1}(G, \mathcal{O}(X) \otimes \chi)=0$ for every characters $\chi$ of $G$. On the other hand, 
putting $X=G$ it is obvious that every affine $G$-bundle $\pi: G \times \mathbb{A}^{1} \rightarrow G$ has a $G$-equivariant section. Hence we get

$$
H_{\text {alg }}^{1}(G, \mathcal{O}(G) \otimes \chi)=0
$$

for every algebraic group $G$ and every character $\chi$. If $G$ is linearly reductive then $\mathcal{O}(G)$ contains every irreducible (rational) representation of $G$ as a direct summand and so $H_{\text {alg }}^{1}(G, M)=0$ for every $G$-module $M$.

Remark 3. It is clear from the proofs above that Proposition 1 and its Corollary 1 hold for an arbitrary ground field $k$, i.e., if everything is defined over $k$.

\section{$\S 2$ Holomorphic affine bundles}

In the following paragraphs we are working over the base field $\mathbb{C}$ of complex numbers. Let $G$ be a complex reductive group and $K \subset G$ a maximal compact subgroup. The first result is analogous to Proposition 1 of the previous paragraph. Let $X$ be a Stein $G$-space and let $\pi: E \rightarrow X$ be a holomorphic affine bundle over $X$, i.e., the fibers of $E$ are isomorphic to $\mathbb{C}^{n}$ for some $n$ and the structure group is the affine group $\operatorname{Aff}\left(\mathbb{C}^{n}\right)$. The following result is an easy consequence of the equivariant version of Oka's principle in [HK95]. It generalizes Theorem 1.2 in [AR95].

Proposition 2. Assume that $X$ is (continuously) $K$-equivariantly retractable to a $K$-fixed point $x_{0} \in X$. Then every lift of the $G$-action to $E$ is holomorphically equivalent to a trivial lift, i.e., the bundle is trivial and the action on the fiber is given by a fixed representation $\tau: G \rightarrow \operatorname{Aff}\left(\mathbb{C}^{n}\right)$ independent of the base point.

Proof. We shortly indicate how to get the result from [HK95]. Let $\tau: G \rightarrow$ $\operatorname{Aff}\left(\mathbb{C}^{n}\right)$ be the representation given by the $G$-action on the fiber $E_{x_{0}}$ of $E$ over the fixed point $x_{0}$ and denote by $E_{0}$ the $G$-product bundle $X \times \mathbb{C}^{n}$ with respect to $\tau$, i.e., the $G$-action on $E_{0}$ is diagonal and on the second factor given by the representation $\tau$. Now consider the $G$-bundle $\operatorname{Iso}\left(E_{0}, E\right)$ ([HK95, p. 340]). Using an equivariant version of the Covering Homotopy Theorem together with the fact that $X$ is $K$-equivariantly retractable to a point one shows that $\operatorname{Iso}\left(E_{0}, E\right)$ admits a topological $K$-equivariant section (see [HK95, §12, Corollary 1, Remark]). Then the Homotopy Theorem in loc. cit. $\S 11$ gives a holomorphic $G$-equivariant section of $\operatorname{Iso}\left(E_{0}, E\right)$ which implies that $E_{0}$ and $E$ are holomorphically $G$-equivariantly isomorphic. 
Let us denote by $\mathfrak{J}_{n}^{\text {hol }}$ the holomorphic Jonquière group:

$$
\begin{array}{r}
\mathfrak{J}_{n}^{\text {hol }}:=\left\{\varphi=\left(\varphi_{1}, \varphi_{2}, \ldots, \varphi_{n}\right) \in \operatorname{Aut}_{h o l}\left(\mathbb{C}^{n}\right) \mid \varphi_{i}=\varphi_{i}\left(z_{1}, \ldots, z_{i}\right)\right. \\
\text { for } i=1, \ldots, n\}
\end{array}
$$

where $\operatorname{Aut}_{h o l}\left(\mathbb{C}^{n}\right)$ is the group of holomorphic automorphisms of $\mathbb{C}^{n}$. Using Proposition 2 above we can carry over the linearization result of $\S 1$ (Corollary 1) to the holomorphic setting. In fact, the same proof works here, too. But in order to get the result also for compact groups (and not only for reductive groups) we have to use a result concerning the following problem:

Extension problem. Given a holomorphic action of a compact group $K$ on $\mathbb{C}^{n}$, does it extend to a holomorphic action of the universal complexification $G=K^{\mathbb{C}}$ ?

For Jonquière-type actions it has a positive answer.

Lemma 1. Every action of a compact group $K$ on $\mathbb{C}^{n}$ by Jonquière automorphisms extends to an action of the universal complexification $G=K^{\mathbb{C}}$ by Jonquière automorphisms.

Proof. By induction, we can view $\mathbb{C}^{n}$ as an affine line bundle over $\mathbb{C}^{n-1}$ on which $K$ acts by bundle isomorphisms and assume that the claim holds for the action on $\mathbb{C}^{n-1}$. Now we can apply the following result due to Heinzner and Ianuzzi (see [HI96, §8 Theorem]):

$A$ holomorphic principal $K$-bundle $P \stackrel{\pi}{\rightarrow} X$ with complex structure group $S$ extends to a holomorphic principal $K^{\mathbb{C}}$-bundle $P^{\star} \stackrel{\pi}{\longrightarrow} X^{\star}$.

We use this theorem in the case where $X=X^{\star}=\mathbb{C}^{n-1}, S=\operatorname{Aff}(\mathbb{C})$ and $P$ is the corresponding (trivial) principal bundle $\mathbb{C}^{n-1} \times \operatorname{Aff}(\mathbb{C}) \rightarrow \mathbb{C}^{n-1}$. Thus the $K$-action on the line bundle $P$ also extends to a $K^{\mathbb{C}}$-action by bundle isomorphisms, and the claim follows.

Corollary 2. Every action of a compact group $K$ or a complex reductive group $G$ on $\mathbb{C}^{n}$ by holomorphic Jonquière automorphisms is linearizable.

\section{$\S 3$. Holomorphic actions by overshears}

In case of dimension 2 we can prove more. Denote by $\mathfrak{S h}_{n}$ the so-called group of overshears. (This notion is introduced in [RR88].) This is the subgroup of Aut ${ }_{h o l}\left(\mathbb{C}^{n}\right)$ generated by affine automorphisms and automorphisms of the form

$$
\left(z_{1}, \ldots, z_{n}\right) \mapsto\left(a\left(z_{2}, \ldots, z_{n}\right) z_{1}+b\left(z_{2}, \ldots, z_{n}\right), z_{2}, \ldots, z_{n}\right)
$$

where $a, b$ are arbitrary holomorphic functions on $\mathbb{C}^{n}$ and $a$ is invertible. Clearly, $\mathfrak{S h}_{n}$ contains the holomorphic Jonquière subgroup $\mathfrak{J}_{n}^{\text {hol }}$. 
Proposition 3. Every holomorphic action of $K($ or $G)$ on $\mathbb{C}^{2}$ by overshears is linearizable, i.e., the image of $K($ or $G)$ in $\mathfrak{S h}_{2}$ is conjugate to a subgroup of $\mathrm{GL}_{2}(\mathbb{C})$.

Remark 4. The group of overshears is dense in the holomorphic automorphism group with respect to the compact-open topology, but it is a proper subgroup of the holomorphic automorphism group ([AL92]). Clearly, compact-open topology is not the right topology for such global questions like linearization. At the end of the paper we will describe an example of a holomorphic automorphism of $\mathbb{C}^{2}$ which is not contained in the group of overshears (cf. loc. cit.).

The proof of the proposition above is based on the following structure theorem due to Ahern and Rudin (see [AR95, Theorem 1.4]).

Theorem. The group $\mathfrak{S h}_{2}$ of overshears on $\mathbb{C}^{2}$ is a free amalgamated product of the affine group $\mathrm{Aff}\left(\mathbb{C}^{2}\right)$ and the holomorphic Jonquière group $\mathfrak{J}_{2}^{\text {hol }}$ over their intersection.

Proof of Proposition 3. By assumption, the action of $K$ is given by a homomorphism $K \rightarrow \mathfrak{S h}_{2}$. The theorem above together with the following Lemma 2 implies that the image of $K$ is conjugate to a subgroup of $\operatorname{Aff}\left(\mathbb{C}^{2}\right)$ or of $\mathfrak{J}_{2}^{\text {hol }}$. In the first case it is well-known that every compact subgroup of $\operatorname{Aff}\left(\mathbb{C}^{2}\right)$ is conjugate to a subgroup of $\mathrm{GL}_{2}(\mathbb{C})$. In the second case we can apply Corollary 2. The case of a holomorphic action of $G$ is clear since $G$ is the universal complexification of any maximal compact subgroup $K$.

Lemma 2. Let $G$ be a topological group which is a free amalgamated product of two closed subgroups $A, B \subset G$ over their intersection $A \cap B$. Then every compact subgroup $K$ of $G$ can be conjugated into one of the two factors.

Proof. We use the following two facts about amalgamated products:

(a) If an element $g \in G$ has roots of arbitrary degree then $g$ can be conjugated into one of the two factors. (This is easy to prove.)

(b) A subgroup $H$ of $G$ of bounded length (i.e. there is an $N \in \mathbb{N}$ such that the length of every element $h \in H$ in the product representation is $\leq N$ ) can be conjugated into one of the two factors (Serre [Se80, I.4.3 Theorem 8]).

We will show that $K$ is of bounded length which implies the claim by fact (b) above.

(1) First suppose that $K$ is a compact torus $T=\left(S^{1}\right)^{k}$. Let $t \in T$ be a regular element which means that the subgroup $\left\{t^{n} \mid n \in \mathbb{Z}\right\}$ is dense in 
$K$. Since $t$ has roots of arbitrary degree it can be conjugated into one of the two factors: $g t g^{-1} \in A$ or $B$ for some $g \in G$, by fact (a) above. Since $t$ is regular and the factors are closed in $G$ we get $g T g^{-1} \subset A$ or $B$. Hence, the length of each element in the torus is at most $2 \times \operatorname{length}(g)+1$.

(2) Next we suppose $K$ to be connected. One can find finitely many compact subtori $T_{1}, T_{2}, \ldots, T_{n} \subset K$ (not necessary mutually disjoint) in $K$ such that the product map $T_{1} \times T_{2} \times \cdots \times T_{n} \rightarrow K$ is surjective. In fact, choose tori $T_{1}, T_{2}, \ldots, T_{k} \subset K$ such that their Lie algebras span the Lie algebra of $K$ as a vector space. Then the differential of the product map at the identity $e \in K$ has maximal rank. Hence, the image of the map contains some neighborhood $V$ of $e \in K$. It is well-known that $V$ generates $K$, hence $K=\bigcup_{i=1}^{\infty} V^{i}$. Since $K$ is compact we have $K=V^{m}$ for some $m \in \mathbb{N}$ and so the product map

$$
\underbrace{\left(T_{1} \times T_{2} \times \cdots \times T_{k}\right) \times\left(T_{1} \times T_{2} \times \cdots \times T_{k}\right) \times \cdots \times\left(T_{1} \times \cdots \times T_{k}\right)}_{k \times m \text { factors }} \rightarrow K
$$

is surjective. Since, by (1), the elements in the tori $T_{i}$ have bounded length the same holds for $K$.

(3) For the general case we remark that a compact group has only finitely many connected components and that each element in a component can be written as a product of a fixed element in that component with an element of the identity component.

Remark 5. In the algebraic setting one knows that Aut $\left(\mathbb{C}^{2}\right)$ is the free amalgamated product of $\operatorname{Aff}\left(\mathbb{C}^{2}\right)$ and $\mathfrak{J}_{2}$ over their intersection (Theorem of van der Kulk [vdK53], cf. [Kr89, §1]). It was shown by Wright in [Wr79] that for an algebraic action of a linear algebraic group $G$ on $\mathbb{C}^{2}$ the image of $G$ in $\operatorname{Aut}\left(\mathbb{C}^{2}\right)$ is of bounded length, hence conjugate to a subgroup of $\operatorname{Aff}\left(\mathbb{C}^{2}\right)$ or of $\mathfrak{J}_{2}$ by Serre's result (see fact (b) of the proof above). An alternate proof of this can be obtained along the lines of the proof above, since every connected linear algebraic group is generated by finitely many subgroups of the form $\mathbb{C}^{*}\left(=\mathrm{GL}_{1}(\mathbb{C})\right)$ and $\mathbb{C}^{+}$, and both groups contain elements $t$ which generate a Zariski-dense subgroup and admit roots of arbitrary degree.

Finally, we mention that Proposition 3 includes a result concerning the Extension Problem (§2):

Corollary 3. Every action of a compact group $K$ on $\mathbb{C}^{2}$ by overshears extends to an action of the universal complexification $G=K^{\mathbb{C}}$ by overshears.

The following example is due to Andersen-Lempert [AL92]. We give a short proof based on our considerations above. 
Example. The holomorphic automorphism $\varphi(z, w)=\left(z e^{z w}, w e^{-z w}\right)$ of $\mathbb{C}^{2}$ is not contained in $\mathfrak{S h}_{2}$.

Proof. Suppose that all the roots $\varphi_{n}(z, w)=\left(z e^{\frac{1}{n} z w}, w e^{-\frac{1}{n} z w}\right)$ of $\varphi$ are contained in $\mathfrak{S h}_{2}$. Then, by fact (a) in the proof of Lemma 2 the automorphism $\varphi$ is conjugated to an automorphism in $\operatorname{Aff}\left(\mathbb{C}^{2}\right)$ or to an automorphism in $\mathfrak{J}_{2}^{\text {hol }}$. Clearly, the fixed point set of an affine automorphism is connected, and it is not difficult to see that the fixed point set of an automorphism in $\mathfrak{J}_{2}^{\text {hol }}$ is either connected or consists of (countably many) copies of $\mathbb{C}$. On the other hand the fixed point set of $\varphi$ consists of copies of $\mathbb{C}^{*}$ together with the coordinate axes. This contradiction shows that one of the roots $\varphi_{n}$ is not contained in $\mathfrak{S h}_{2}$. Since $\varphi$ and $\varphi_{n}$ are conjugated by a linear map the claim follows.

Note added in Proof: The second author and Harm Derksen have recently shown that there exist non-linearizable holomorphic actions of $\mathbb{C}^{*}$ on affine 4 -space $\mathbb{C}^{4}$. The details will appear elsewhere.

\section{References}

[AL92] E. Andersen and L. Lempert, On the group of holomorphic automorphism of $\mathbb{C}^{n}$, Invent. Math. 110 (1992), 371-388.

[AR95] P. Ahern and W. Rudin, Periodic automorphisms of $\mathbb{C}^{n}$, Indiana Univ. Math. J. 44 (1995), 287-303.

[HI96] P. Heinzner and A. Ianuzzi, Integration of local actions on holomorphic fibre spaces, Nagoya Math. J. (1996) (to appear).

[HK95] P. Heinzner and F. Kutzschebauch, An equivariant version of Grauert's Oka principle, Invent. Math. 119 (1995), 317-346.

[Iv96] N. Ivanenko, Some classes of linearizable polynomial maps, J. Pure Appl. Algebra (1996) (to appear).

[KK89] F. Knop, H. Kraft, and Th. Vust, The Picard group of a G-variety, Algebraische Transformationsgruppen und Invariantentheorie (H. Kraft, P. Slodowy, T. A. Springer, eds.), DMV-Seminar, vol. 13, Birkhäuser Verlag, BaselBoston, 1989, pp. 77-87.

[Kr89] H. Kraft, Algebraic automorphisms of affine space, Topological methods in algebraic transformation groups (H. Kraft, T. Petrie, G.W. Schwarz, eds.), Progress in Math., vol. 80, Birkhäuser Verlag, Basel-Boston, 1989, pp. 81105.

[RR88] J.-P. Rosay and W. Rudin, Holomorphic maps from $\mathbb{C}^{n}$ to $\mathbb{C}^{n}$, Trans. Amer. Math. Soc. 310 (1988), 47-86. 
[Se80] J.-P. Serre, Trees, Springer Verlag, Berlin-Heidelberg-New York, 1980.

[vdK53] van der Kulk, On polynomial rings in two variables, Nieuw Arch. Wisk. 1 (1953), 33-41.

[Wr79] D. Wright, Abelian subgroups of Aut $_{k}(k[x, y])$ and applications to actions on the affine plane, Illinois J. Math. 23 (1979), 579-634.

M athematisches Institut, Universität Basel, Rheinsprung 21, CH-4051 B A SEL, SW IT Z E R L A N D

E-mail address: kraft@math.unibas.ch, kutzsche@math.unibas.ch 